\title{
DISTRIBUCIÓN GEOGRÁFICA DE PHYLLARIOPSIS PURPURASCENS (C. AGARDH) HENRY \& SOUTH Y P. BREVIPES (C. AGARDH) HENRY \& SOUTH EN LA PENÍNSULA IBÉRICA E ISLAS BALEARES
} $\rightarrow \infty \infty^{\infty}$

\author{
Antonio FLORES-MOYA, Francisco CONDE y José L. IZQUIERDO
}

\begin{abstract}
RESUMEN. Distribución geográfica de Phyllariopsis purpurascens (C. Agardh) Henry \& South y P. brevipes (C. Agardh) Henry \& South en la Península Ibérica e Islas Baleares. Se presentan los mapas de distribución en la Península Ibérica e Islas Baleares de Phyllariopsis purpurascens. (C. Agardh) Henry \& South y P. brevipes (C. Agardh) Henry \& South (Phyllariaceae, Laminariales, Phaeophyta), y se comentan sus respectivos rangos verticales de distribución. Se discuten las consecuencias de la distribución geográfica del género Phyllariopsis, en relación a su diversificación ecológica y reproductiva.
\end{abstract}

Palabras clave. Distribución, mapa, Phyllariopsis, Península Ibérica, algas marinas.

ABSTRACT. Geographical distribution of Phyllariopsis purpurascens (C. Agardh) Henry \& South and P. brevipes (C. Agardh) Henry \& South in the Iberian Peninsula and Balearic Islands. The distribution maps of Phyllariopsis purpurascens (C. Agardh) Henry \& South and P. brevipes (C. Agardh) Henry \& South (Phyllariaceae, Laminariales, Phaeophyta), along the Iberian Peninsula and Balearic Islands, are presented, and their vertical distribution range are commented. The consequences of the geographical distribution in Phyllariopsis, in relation to their ecological and reproductive diversification, are discussed.

Key words. Distribution, map, Phyllariopsis, Iberian Peninsula, seaweeds.

\section{INTRODUCCIÓN}

El conocimiento de la distribución geográfica de las especies incluidas en Phyllariopsis Henry \& South (Phyllariaceae, Laminariales, Phaeophyta) es fragmentario, en gran parte debido a la escasa cantidad de material de herbario. Henry \& South (1987), en un trabajo donde revalidaron la familia Phyllariaceae, presentaron un bosquejo general de la corología de las especies incluidas en ella. Pérez-Cirera et al. (1991) describieron la distribución de Phyllariopsis en el Atlántico europeo (que sólamente concierne a los litorales de la Península Ibérica). Ribera et al.(1992) presentaron una extensa "check-list" de las Phaeophyta del Mediterráneo, donde confirman la presencia de Phyllariopsis brevipes (C. Ag.) Henry \& South y Phyllariopsis purpurascens (C. Ag.) Henry \& South en las costas españolas; no obstante, para esta segunda especie sólamente recogen la referencia de 
Conde (1984) de material arrojado para las costas de Málaga.

La familia Phyllariaceae constituye una línea independiente de la vía principal de evolución en Laminariales (Henry \& South, 1987), que bien pudieron originarse en el Pacífico Norte y sobrevivir en el Artico antes de alcanzar el Atlántico, o bien son un grupo relicto autóctono del Atlántico Norte y el Artico adyacente (Lüning, 1990). Esta afirmación se basa en el hecho de que las Laminariales tienen su origen en el Pacífico Norte (Druehl, 1970, 1981b). Esta idea ha sido apoyada con los recientes estudios sobre Pseudochordaceae, la familia que presenta mayor porcentaje de caracteres primitivos en el orden (Kawai \& Kurogi, 1985; Kawai \& Nabata, 1990), con dos especies del Pacífico Norte. La familia Phyllariaceae se restringe al Atlántico y el Mediterráneo adyacente (Henry \& South, 1987; Lüning, 1990), donde otras familias del orden están ausentes (Pseudochordaceae, Chordaceae y Lessoniaceae) o muy dispersas (Alariaceae y Laminariaceae), en comparación con su distribución en el Pacífico Norte (Druehl, 1981b).

En este trabajo se presenta la distribución geográfica del género Phyllariopsis (Phyllariaceae, Laminariales, Phaeophyceae) en la Península Ibérica e Islas Baleares. Sólo se conocen dos especies de este género: $P$. purpurascens y $P$. brevipes (Henry \& South, 1987). De esta última, se ha descrito recientemente una nueva subespecie, $P$. brevipes subsp. pseudopurpurascens Pérez-Cirera, Cremades, Bárbara y López (Pérez-Cirera et al., 1991). Los areales de distribución de los taxa de Phyllariaceae (junto con otros datos de tipo reproductivo y ecológico) serán empleados para explicar la diversificación dentro de la familia.

\section{MATERIALES Y MÉTODOS}

La información utilizada para elaborar los mapas de distribución geográfica y deter- minar los rangos verticales de distribución procede de ejemplares conservados en los herbarios de Aurelio Aranda (Institut d'Ecologia Litoral, El Campello, Alicante), BCF-A, ITAC (Laboratorio de Ficología, Facultad de Biología, Universidad Complutense de Madrid) MGC-Phyc y VAB, así como de las referencias bibliográficas consultadas. El procedimiento seguido para la elaboración de los mapas es similar al empleado por Margalet y Navarro (1990). Se han distinguido tres tipos de testigos para los mapas de distribución (figs 1-3): ejemplares recogidos in situ, ejemplares procedentes de arribazón y citas bibliográficas. En todos los casos, se ha caracterizado la localidad de la muestra mediante cuadrícula del sistema UTM de $10 \mathrm{~km}$ de lado.

\section{RESULTADOS Y DISCUSIÓN}

$P$. purpurascens se distribuye en la $\mathrm{Pe}$ nínsula Ibérica por la fachada atlántica de Galicia y norte de Portugal, zona del Estrecho de Gibraltar y Mar de Alborán, llegando hacia el este hasta las Islas Hormigas (Murcia) (fig. 1). Se han encontrado ejemplares arrojados en la costa del Algarve (Ardré, 1969) y en el litoral de Cantabria; por lo tanto, es posible que el areal de esta especie sea mayor, aunque habría que confirmarlo con material recolectado in situ. Otras áreas, diferentes a la Península Ibérica, donde se ha descrito la presencia de esta especie son: costas de Melilla, Argelia, Estrecho de Messina, Cerdeña (Ribera et al., 1992) y noroeste de Marruecos (Feldmann, 1934).

$P$. brevipes subsp. brevipes se encuentra en las costas de Asturias y, desde los alrededores de Lisboa, se distribuye de forma casi continua por el sur de Portugal y las costas mediterráneas de la Península Ibérica (fig. 2). Este taxon también se ha citado, para la zona atlántica, en las costas del Golfo de Vizcaya (donde estaría su límite septentrional) y no- 


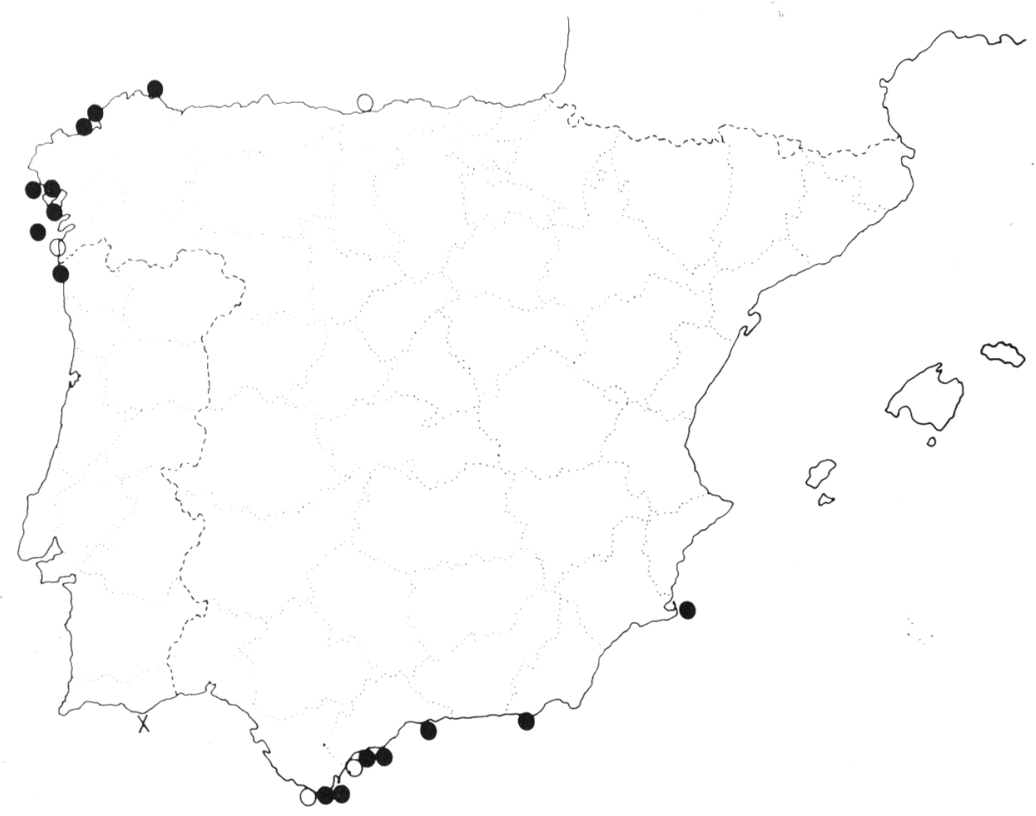

Figura 1. Distribución geográfica de Phyllariopsis purpurascens en la Península Ibérica e Islas Baleares. Material recogido in situ o dragado $(\bullet)$, material de arribazón (o) o cita bibliográfica (x). Distribution of Phyllariopsis purpurascens along the Iberian Peninsula and Balearic Islands. Samples collected in situ or by dragage $(\bullet)$, drift $(\mathrm{o})$, or references $(\mathrm{x})$.

roeste de Marruecos (Feldmann, 1934). En el Mediterráneo se ha descrito su presencia en los Pirineos Orientales (Francia), Córcega, Estrecho de Messina, Argelia y Marruecos (Ribera et al., 1992; González-García y Conde, 1993). $P$. brevipes subsp. pseudopurpurascens se encuentra restringida, según los ejemplares estudiados, a las costas de Galicia y Minho, y a los alrededores de Lisboa (fig. 3).

La escasa cantidad de pliegos es el principal problema para abordar el estudio corológico de estos taxones; evidentemente, ello se debe a que estas especies suelen aparecer siempre en profundidad, por debajo de $25 \mathrm{~m}$ en el caso de $P$. purpurascens (Giaccone, 1969; Flores-Moya et al., en prensa) y a profundidades aún mayores, de varias decenas de metros, en el caso de $P$. brevipes subsp. brevipes.(Ballesteros, 1984; Henry, 1987b; Soto, 1987; Boisset, 1991; González-García y Conde, 1993). No obstante, se han encontrado poblaciones intermareales (si se puede aplicar este término al Mediterráneo) de este último taxon en el Estrecho de Messina (Henry, com. pers.). Por lo tanto, la delimitación del área de distribución oscila entre las "pruebas de ausencia" y la "ausencia de pruebas" (Druehl, 1981a: 306). El uso extendido de la escafandra autónoma permitirá un mejor conocimiento de la distribución geográfica de estas especies.

El rango de profundidades osciló entre 4 y $60 \mathrm{~m}$ de profundidad para $P$. purpurascens, aunque la gran mayoría corresponden a más de $20 \mathrm{~m}$. La presencia del alga a $4 \mathrm{~m}$ (Conde et al., 1990) en Estepona (Málaga) debe considerarse un hecho extraordinario, ligado a las especiales características oceanográficas de esta zona, con fuertes corrientes y donde existen surgencias de aguas profundas frías (Rodríguez, 1981). Este fenómeno podría denominarse "emersión isotérmica", en contraposición al de "inmersión isotérmica", empleado por Briggs (1974) para explicar la presencia en profundidad de especies de superficie sensi- 


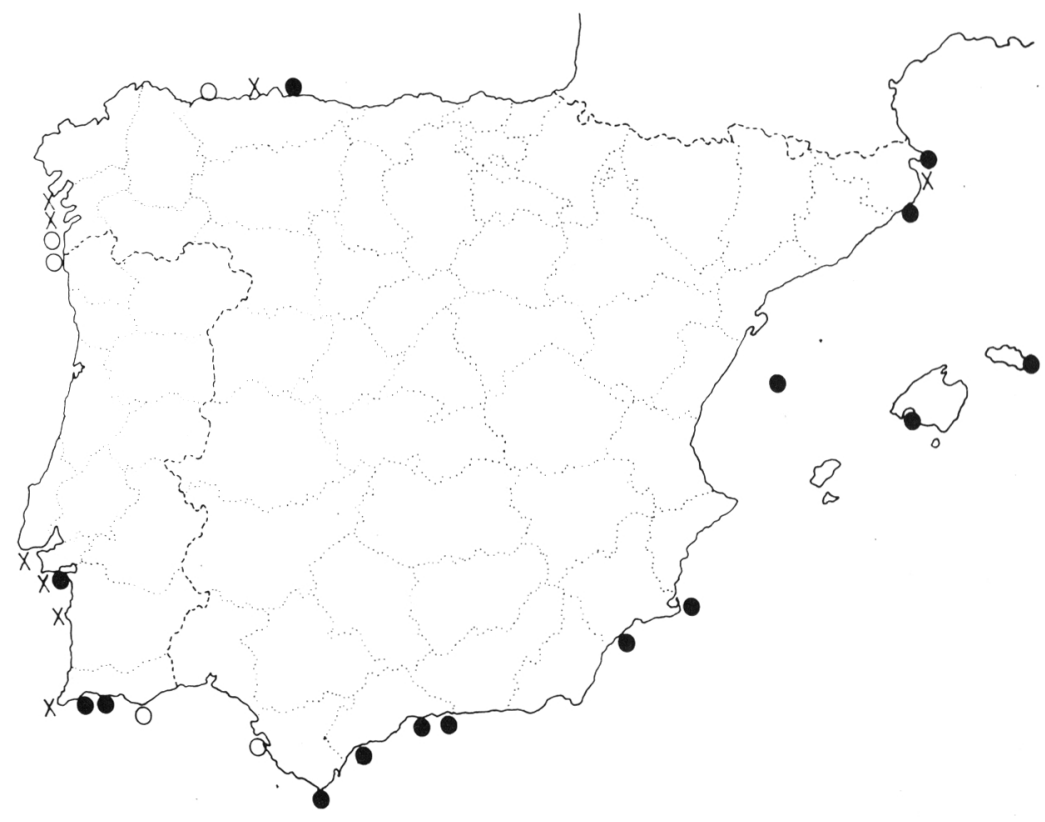

Figura 2. Distribución geográfica de Phyllariopsis brevipes subsp. brevipes en la Península Ibérica e Islas Baleares. Material recogido in situ o dragado (•), material de arribazón (o) o cita bibliográfica (x). Distribution of Phyllariopsis brevipes subsp. brevipes along the Iberian Peninsula and Balearic Islands. Samples collected in situ or by dragage $(\bullet)$, drift $(\mathrm{o})$, or references $(\mathrm{x})$.

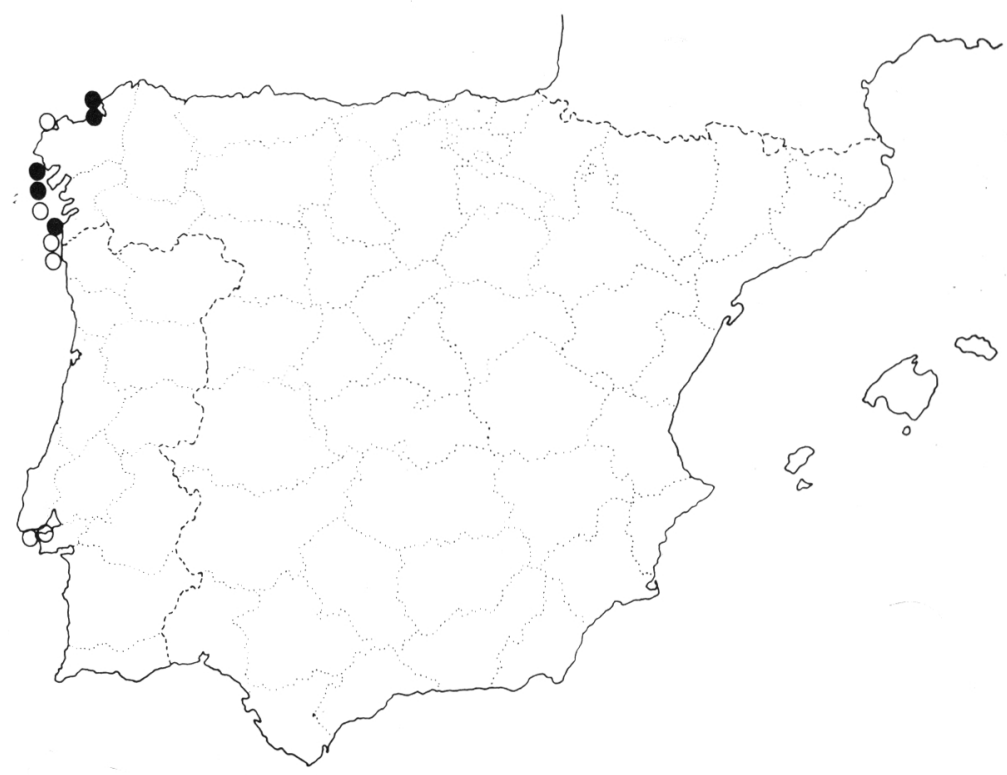

Figura 3. Distribución geográfica de Phyllariopsis brevipes subsp. pseudopurpurascens en la Península Ibérica e Islas Baleares. Material recogido in situ o dragado $(\bullet)$, material de arribazón (o) o cita bibliográfica (x). Distribution of Phyllariopsis purpurascens (C. Ag.) Henry \& South along the Iberian Peninsula and Balearic Islands. Samples collected in situ or by dragage $(\bullet)$, drift (o), or references (x). 
bles a altas temperaturas.

$P$. brevipes subsp. brevipes se presenta en un amplio rango vertical, desde pocos metros de profundidad en la zona atlántica de Galicia (Pérez-Cirera et al., 1991) y el Mar de Alborán, hasta varias decenas de metros en la zona levantina-balear (Ballesteros, 1984; Soto, 1987; Boisset, 1991). Las poblaciones profundas del Mediterráneo se explicarían como un fenómeno de «inmersión isotérmica» (Briggs, 1974). P. brevipes subsp.pseudopurpurascens se presenta a muy poca profundidad (PérezCirera et al., 1991).

Es de destacar que algunos ejemplares de $P$. brevipes subsp. brevipes de la región mediterránea no se ajustan a las características dadas por Pérez-Cirera et al. (1991) para el litoral atlántico. Por ejemplo, según estos autores, el estipe es corto o nulo, nunca mayor de $2 \mathrm{~cm}$ de longitud, para este taxon, mientras que en $P$. brevipes subsp. pseudopurpurascens la longitud oscila entre (2) 4-9 (13) cm. La longitud del estipe de $P$. brevipes subsp. brevipes en las Islas Hormigas (herbario de Aurelio Aranda, 692 y 693) supera los $5 \mathrm{~cm}$; un tamaño similar se ha encontrado en ejemplares del litoral mediterráneo marroquí (González-García y Conde, 1993: MGC-Phyc 2165). Es obvio que este carácter no es aplicable para las poblaciones del Mediterráneo, siendo necesario un estudio más amplio, con muestras de todo el areal de distribución, con el objeto de establecer buenos caracteres diferenciales entre ambas subespecies.

Las distribuciones geográficas de cada una de las especies de la familia Phyllariaceae (para el género Saccorhiza de la Pyl., ver Lüning, 1990: 51 y 59; para el género Phyllariopsis, Henry \& South, 1987, y este trabajo) permiten suponer que el género Saccorhiza ha tenido una diversificación ecológica y morfológica mayor que el género Phyllariopsis. Saccorhiza dermatodea (de la Pyl.) J. Ag. tiene una distribución árticanoratlántica, Saccorhiza polyschides
(Lighfoot) Batters habita en las costas europeas y norteafricanas del atlántico (desde Noruega hasta Cabo Jubi, Marruecos) y Mediterráneo adyacente, y ambas especies del género Phyllariopsis se restringen a las costas atlánticas de la Península Ibérica y norte de Marruecos y la zona adyacente del mar Mediterráneo. Esta idea también es congruente con las características exhibidas por las fases gametofíticas: $S$. dermatodea es monoica (Henry, 1987a), S. polyschides es dioica y dimórfica (Sauvageau, 1918), mientras que ambas especies de Phyllariopsis son dioicas pero monomórficas (Henry, 1987b; FloresMoya et al., en prep.). Por último, la descripción recientemente de Phyllariopsis brevipes subsp. pseudopurpurascens (Pérez-Cirera et al., 1991), cuyos gametófitos son desconocidos, permite suponer que la diversificación del género Phyllariopsis no ha sido tan intensa como en Saccorhiza.

\section{APÉNDICE}

Phyllariopsis purpurascens (C. Agardh) Henry \& South

CANTABRIA. 30TUP90: «Ría de Tina Mayor, Pechón», T. Almaraz 18-VII-1992, ITAC 2364; arrojada.

LUGO. 29TPJ23: «Cabo San Ciprián» (Polo et al., 1979 y Gili et al., 1979 in Pérez-Cirera et al., 1991); de -18 a $-30 \mathrm{~m}$.

LA CORUÑA. Todas las citas de La Coruña pertenecen a la publicación de Pérez-Cirera et al. (1991). 29TNJ40: «Dique de abrigo Barrié de la Maza», J. Cremades y I. Bárbara 16-X-1986, SANTAlgae 1345; de -9 a -13 m. Ibídem, J. Cremades y I. Bárbara 30-XI-1986, SANT-Algae 1346. «San Amaro», J. Cremades y I. Bárbara 07-XI-1986, SANT-Algae 1347. «Riazor», F. Bescansa 17-X1914; arrojada. «Riazor», F. Bescansa 1-XI-1916. «Orzán», F. Bescansa VI-1915; dragado. «La Coruña», F. Bescansa. 29TNJ51: «Cabo Prioriño, ría de El Ferrol», J. Cremades y I. Bárbara 18-VII1989, SANT-Algae 951; arrojada. «Punta Fornelos, 
ría de El Ferrol», J. Cremades y I. Bárbara 07-VII1986, SANT-Algae 1344; a -9 m. Localidades a las que no se ha podido asignar referencia UTM: Sauvageau (1897, in Ardré, 1969).

PONTEVEDRA. Todas las citas de Pontevedra pertenecen a la publicación de PérezCirera et al. (1991). 29TNG07: «Islas Cies, ría de Vigo», J. Otero 04-VIII-1989, SANT-Algae 1808; de -15 a -17 m. 29TNG09: «Islas Ons», F. Miranda 19-V-1933. 29TNG16: «Punta Sansón, Bayona», F. Bescansa 05-IX-1914; arrojada. 29TNG18: «Bueu», M. C. López 29-VI-1991, SANT-Algae 3004; arrojada. «Aguete», F. Miranda 4-VI-1933; dragado a 12-14 $\mathrm{m}$ de profundidad. 29TNG19: «Punta Fentiñanzo», F. Miranda 27-V-1933, dragado a $18-20 \mathrm{~m}$ de profundidad.

MINHO. 29TNG11: «Viana do Castelo», T. Gallardo y M. J. Navarro 23-VI-1989, ITAC 2364; fija.

ALGARVE. 29SNA99: «Faro», R. Lami IX1933; arrojada (Pérez-Cirera et al., 1991). «Faro», J. Feldmann 14-VIII-1960, arrojada (Ardré, 1969).

CÁDIZ. 30STE69: «Tarifa: camping de Tarifa», J. J. Vera y F. Conde 06-X-1990, MGC-Phyc 2056; arrojada. 30STE68: «Tarifa», desde IV a X1991; -30 m (Flores-Moya et al., en prensa). 30STE89: «Algeciras: Punta Carnero», F. Conde 13-V-1983, MGC-Phyc 1210. Localidades a las que no se ha podido asignar referencia UTM: Agardh (1823), holótipo, (ver Henry \& South, 1987).

MÁLAGA. 30SUF03: «Estepona», F. Conde 28-I-80, MGC-Phyc 604; arrojada. «Estepona», J. A. Fernández 03-VII-1981, MGC-Phyc 744; arrojada. 30SUF13: «Estepona: camping La Chimenea», A. Flores 22-X-1989, MGC-Phyc 1861; a -4 m. «Estepona: camping La Chimenea», A. Flores y A. Giráldez 10-XII-1989, MGC-Phyc 1862; arrojada (Conde et al., 1990). «Estepona» desde IV a X-1990, a -30 m (Flores-Moya et al., en prensa). 30SUF23: «San Pedro de Alcántara: Placer de las Bóvedas», F. Conde 06-VI-1978, MGC-Phyc 603; arrojada. «Marbella: Puerto Banús», A. Flores 11VI-92, MGC-Phyc 2148; dragado a $-20 \mathrm{~m}$. «Marbella: Puerto Banús», A. Flores y R. Sierra 17-IX-1992, MGC-Phyc 2118; dragado a $-20 \mathrm{~m}$. 30SUF86: «Chilches», A. Flores y A. Barrajón 16I-1993, MGC-Phyc 2152; dragado a -60 m.

ALMERÍA. 30SWF06: «Adra: castillo de Guardias Viejas», A. Flores 05-XI-1990, MGCPhyc 2112; dragado a $-35 \mathrm{~m}$.
MURCIA. 30SYG07: «Islas Hormigas», A. Ramos 12-X-1991, herbario de A. Aranda 691; entre 40 y $48 \mathrm{~m}$ de profundidad.

Phyllariopsis brevipes (C. Agardh) Henry \& South subsp. brevipes

ASTURIAS. 30TTP82: «Gijón», fija (Sauvageau, 1918 in Pérez-Cirera et al., 1991). 30TTP52: «Salinas de Avilés» (Lázaro Ibiza, 1900 in Pérez-Cirera et al., 1991). 29TPJ82: «Playa de Navia, margen derecha», T. Gallardo y M. Alvarez 13-VII-1988, ITAC 1635; arrojada.

PONTEVEDRA. 29TNG18: «Ría de Pontevedra» (Niell, 1978). 29TNG17: «Ría de Vigo» (Niell, 1978). 29TNG16: «Bayona», arrojada (Hamel, 1928). 29TNG13: «La Guardia», arrojada (Hamel, 1928).

ESTREMADURA. 29SMC68: «Parede» (Ardré, 1969). 29SMC95: «Sesimbra» (Ardré, 1969). 29SNC05: «Arrabida» (Ardré, 1969). «Portinho de Arrabida», Welwitsch 1-VII-1852, LISU P50287, P50280; fija (Pérez-Cirera et al., 1991).

BAJO ALENTEJO. 29SNC10: «Sines» (Ardré, 1969).

ALGARVE. 29SNA09: «Sagres» (Ardré, 1969). 29SNB20: «Lagos», R. Lami 17-IX-1932, COI; fija (Pérez-Cirera et al., 1991). 29SNB40: «Praia da Rocha» (Ardré, 1969). «Praia de Carvoeiro», fija (Palminha, 1957). «Carvoeiro» (Ardré, 1969). 29SNA99: «Faro», Welwitsch V1947, LISU P50281, P50282 y s.n.; arrojada (PérezCirera et al., 1991).

CÁDIZ. 30STE68: «Tarifa», J. Seoane V1961, BCF-A 560. «Tarifa», fija (Seoane, 1965). «Tarifa», 17-V-1988, BCF-A 558; fija. Localidades a las que no se ha podido asignar referencia UTM: Cabrera, holótipo, (ver Henry \& South, 1987).

MÁLAGA. 30SUF03: «Estepona», 24-VIII1988, BCF-A 556; recogida en arrastre. 30SUF86: «Frente estación de Chilches», A. Barrajón 3-VIII1993, MGC-Phyc 2192; -8 m. Localidades a las que no se ha podido asignar referencia UTM: Simón de Rojas Clemente, Herbario de la Sociedad Malagueña de Ciencias, actualmente en GDA (PérezCirera et al., 1991).

MURCIA. 30SXG24: «Aguilas», dragaje a 30-40 m. (Soto, 1987). 30SYG07: «Islas Hormi- 
gas», A. Ramos 12-X-1991, herbario de A. Aranda 692 y 693 ; entre 40 y $48 \mathrm{~m}$ de profundidad.

CASTELLÓN. 31SCE01: «Piedra Joaquín, Islas Columbretes», F. Boisset 6-VII-1985, VAB 00542; a -30 m. «La Horadada, Islas Columbretes», VI-1977, ITAC 2670. «Piedra Joaquín, Islas Columbretes», entre -20 y -35 m (Boisset, 1991).

GERONA. 31TDG91: «Tossa», a -51 m (Ballesteros, 1984). «Isla de Tossa», a -18 m. (Ballesteros, 1984). «Santa Bárbara», a -47 m. (Ballesteros, 1984). 31TEG15: «Islas Medas» (Polo et al., 1982). «Islas Medas» (Ballesteros et al., 1984). 31TEG27: «Cabo Norfeo», a -20 m. (Perera, 1987).

BALEARES. 31SDD77: «Boca de la Bahía de Palma», a -50 m. (Buen, 1916 in Ribera, 1983). 31SFE11: «Al SE del puerto de Mahón», de -45 a -130 m. (Rodríguez, 1889 in Ribera, 1983). «Hacia los Freus», J.J. Rodríguez 28-X-1889, MA-Algae 0365; dragada a -55 m. (Pérez-Cirera et al., 1991).

Phyllariopsis brevipes (C. Agardh) Henry \& South subsp. pseudopurpurascens PérezCirera, Cremades, Bárbara y López

LA CORUÑA. Todas las citas de La Coruña pertenecen a la publicación de Pérez-Cirera $e t$ al. (1991). 29TNJ51: «Cabo Prioriño, Ría de El Ferrol», J. Cremades y I. Bárbara 18-VIII-1989, SANT-Algae 1807; a -2 m. Ibídem, J. Cremades y I. Bárbara 23-VIII-1990, SANT-Algae 903. 29TNJ50: «Bahía de La Coruña, Ensenada de Canabal», I. Bárbara 25-VII-1990, SANT-Algae 2505; a 0 m. Ibídem, J. Cremades y I. Bárbara 13VIII-1990, SANT-Algae 2539; a -4 m. «Bahía de La Coruña, al este de Isla Castelo», J. Cremades y I. Bárbara 3-VII-1988, SANT-Algae 1802; arrojada. 29TMH87: «Playa de Muxía, Ría de Camariñas», J. Cremades y I. Bárbara 1-VI-1988, SANT-Algae 1801; arrojada. 29TMH93: «Punta Lens, Muros, Ría de Muros y Noia», J. Cremades 10-VI-1989, SANT-Algae 1803; de 0 a -5 m. «Punta Queixal, Muros, Ría de Muros y Noia», J. Cremades 20-V-1989, SANT-Algae 1804; a -2 m. 29TMH90: «Isla de Sálvora, Ría de Arosa», F. Miranda 11-VI-1934; arrojada. 29TMH92: «Playa de Xudemil, Queiruga, Ría de Muros y Noia», J. Cremades 3-VI-1990, SANT-Algae 1800; a -1 m.

PONTEVEDRA. 29TNG06: «Al este de Cabo Silleiro», T. Gallardo, T. Almaraz y J. L. Izquierdo 22-VII-1993, ITAC 2671; fija. 29TNG05:
«Mougas, Camping», T. Gallardo, T. Almaraz y J. L. Izquierdo 22-VII-1993, ITAC 2672; arrojada. MINHO. 29TNG12: «Carreço, Montedor», T. Gallardo, T. Almaraz y J. L. Izquierdo 21-VII1993, ITAC 2673; arrojada.

ESTREMADURA. 29SMC78: «Paço d'Arcos», Welwitsch VI-1848, LISU P50288; arrojada (Pérez-Cirera et al., 1991). 29SMC88: «Caçilhas», Welwitsch VII-1844, LISU P50289; arrojada (Pérez-Cirera et al., 1991). «Pedrouços», Welwitsch VII-1844, LISU P50292; arrojada (Pérez-Cirera et al., 1991).

AGRADECIMIENTOS. Este trabajo ha sido subvencionado, en parte, por la CICYT con cargo al proyecto $\mathrm{PB}$ 91-0962, y mediante una beca de FPI de la Junta de Andalucía a A. F.-M. Agradecemos gentilmente la cesión de los pliegos de los herbarios de Aurelio Aranda (Institut d'Ecologia Litoral, El Campello, Alicante), BCF$\mathrm{A}$ y $\mathrm{VAB}$, así como la colaboración prestada en todo momento por el Dr. Tomás Gallardo (Universidad Complutense de Madrid). La ayuda de Agustín Barrajón fue inestimable en las numerosas inmersiones efectuadas por A. F.-M. a lo largo de las costas andaluzas.

\section{BIBLIOGRAFÍA}

AGARDH, C. -1823-Species algarum rite cognitae cum synonymis, differentiis specificis et descriptionibus succintis. Vol. 1. Gryphiswaldiae. $531 \mathrm{pp}$.

ARDRÉ, F. -1969-Contribution à l'étude des algues marines du Portugal. I. La flore. Port. acta biol., B, 10: 137-560.

BALLESTEROS, E. -1984- Contribució al coneixement algològic de la Mediterrània espanyola, V. Collect. Bot. (Barcelona), 5: 5968.

BALLESTEROS, E., LL. POLO y J. ROMERO 1984- Vegetació submarina de les illes Medes. I. Algues. In Els sistemes natural de les illes Medes. ROS, J., I. OLIVELLA y J. M. GILI (eds.). Arxius de la Secció de Ciènces 73, I.E.C., Barcelona, p. 333-371.

BOISSET, F. -1991- Contribución al conocimiento algológico del litoral levantino (España, 
Mediterráneo). III. Lazaroa, 12: 53-68.

BRIGGS, J. C. -1974- Marine zoogeography. McGraw-Hill, New York, 475 pp.

CONDE, F. -1984- Catálogo de las algas macrobentónicas marinas de Málaga. Acta Bot. Malacitana, 9: 47-78.

CONDE, F., A. FLORES-MOYA y J. J. VERA 1990- Notas corológicas del macrofitobentos de Andalucía (España). II. Acta Bot. Malacitana, 15: 350-352.

DRUEHL, L. -1970- The pattern of Laminariales distribution in the northeast Pacific. Phycologia, 9: 237-247.

DRUEHL, L. -1981a- Geographical distribution. In The biology of seaweeds. LOBBAN, C. S. y M. J. WYNNE (eds.). Blackwell Scientific Publications, Oxford, pp. 306-325.

DRUEHL, L. $-1981 \mathrm{~b}$ - The distribution of Laminariales in the North Pacific with reference to environmental influences. In: Evolution today. Proceedings of the Second International Congress of Systematic and Evolutionary Biology. SCCUDER, G.G.E. y REVEAL J.L. (eds.). Hunt Institute for Botanical Documentation, Carnegie-Mellon University, Pittsburgh, pp. 55-67.

FELDMANN, J. -1934- Les laminariacées de la Méditerranée et leur répartition géographique. Bull. Travaux St. d'Aquiculture et de Pêche de Castiglione, Alger, 2: 3-42.

FLORES-MOYA, A., J. A. FERNÁNDEZ y F. X. NIELL -en prensa- Reproductive phenology, growth and primary production of Phyllariopsis purpurascens (Phyllariaceae, Phaeophyta) growing at $30 \mathrm{~m}$ depth in the Straits of Gibraltar area. European Journal of Phycology.

GIACCONE, G. -1969- Note sistematiche ed osservazioni fitosociologiche sulle Laminariales del Mediterraneo occidentale. Giorn. Bot. Ital., 103: 457-474.

GONZÁLEZ-GARCÍA, J. A. y F. CONDE -1993Un estudio biogeografico de las Fucales y Laminariales atlánticas en el litoral mediterráneo de Marruecos. Acta Bot. Malacitana, 18.

HAMEL, G. -1928- Les Algues de Vigo. Rev. Algol., 4: 81-95.

HENRY, E. C. -1987a- Primitive characters and a photoperiodic response in Saccorhiza dermatodea (Laminariales, Phaeophyceae). $\mathrm{Br}$. Phycol. J., 22: 23-31.
HENRY, E. C. -1987b- The life history of Phyllariopsisbrevipes (=Phyllaria reniformis) (Phyllariaceae, Laminariales, Phaeophyceae), a kelp with dioecious but sexually monomorphic gametophytes. Phycologia, 26: 17-22.

HENRY,E.C. y G. R. SOUTH -1987-Phyllariopsis gen. nov. and a reappraisal of the Phyllariaceae Tilden 1935 (Laminariales, Phaeophyceae). Phycologia, 26: 9-16.

KAWAI, H. y M. KUROGI -1985- On the life history of Pseudochorda nagaii (Pseudochordaceae fam. nov.) and its tranfer from Chordariales to the Laminariales (Phaeophyta). Phycologia, 24: 289-296.

KAWAI, H. y S. NABATA -1990- Life history and systematic position of Pseudochorda gracilis sp. nov. (Laminariales, Phaeophyceae). J. Phycol., 26: 721-727.

LÜNING, K. -1990-Seaweeds. Their environment, biogeography, and ecophysiology. John Wiley \& Sons, Inc., New York, 527 pp.

MARGALET, J. L. y M. J. NAVARRO -1990Mapas de distribución de algas marinas de la Península Ibérica. I. Bifurcaria bifurcata R. Ross y Halidrys siliquosa (L.) Lyngb. Bot. Complutensis, 16: 99-107.

NIELL, F.X. - 1978-Catálogo florístico y fenológico de las algas superiores y cianofíceas bentónicas de las Rías Bajas Gallegas. Invest. Pesq., 42: 365-400.

PALMINHA, F. -1957- Contribuçoes para o estudo das algas marinhas portuguesas. II. (Litoral Algarvio). Bol. Soc. Port. Cien. Nat., Ser. 2, 8: 68-74.

PERERA, M. -1987-Resultados de un transecto de la vegetación bentónica del litoral del Cabo Norfeu (Gerona). Actas VI Simp. Nac. Bot. Criptog., p. 99-107.

PÉREZ-CIRERA, J. L., J. CREMADES, I. BÁRBARA y M. C. LÓPEZ -1991- Contribución al conocimiento del género Phyllariopsis (Phyllariaceae, Phaeophyta) en el Atlántico europeo. Nova Acta Científica Compostelana (Bioloxía), 2: 3-11.

POLO, LL., M.L. ROMERO, J. ROMERO y M. MOLINS -1982- Algas bentónicas de las islas Medes. Actas I Simp. Ibér. Estud. Bentos Mar., 1: 37-50.

RIBERA, M.A. -1983-Estudio de la flora bentónica marina de las Islas Baleares. Tesis Doctoral, 
Universidad de Barcelona. Barcelona, $636 \mathrm{pp}$. RIBERA, M. A., A. GÓMEZ-GARRETA, T. GALLARDO, M. CORMACI, G. FURNARI y G. GIACCONE -1992- Check-list of Mediterranean seaweeds. I. Fucophyceae (Warming, 1884). Bot. Mar., 35: 109-130.

RODRIGUEZ, J. -1981- Oceanografía del mar Mediterráneo. Ed. Pirámide, Madrid, 174 pp. SAUVAGEAU, C. -1918- Recherches sur les laminaires des côtes de France. Mém. Acad. Sci. Paris, 56: 1-240.

SEOANE, J.A. -1965- Estudios sobre las algas bentónicas de la costa sur de la Península Ibérica (litoral de Cádiz). Invest. Pesq., 29: 3-216.
SOTO, J. -1987- Estudio florístico, corológico, autoecológico y sinecológico de las algas bentónicas marinas del Sureste de la Península Ibérica. Tesis Doctoral, Universidad de Málaga. Málaga, 507 pp.

Aceptado para su publicación en Julio de 1993

Dirección de los autores.A, Flores y F. Conde: Departamento de Biología Vegetal. Facultad de Ciencias. Universidad de Málaga. Campus de Teatinos s/n. 29071 Málaga. J.L. Izquierdo: Departamento de Biología Vegetal I. Facultad de Biología. Universidad Complutense. 28029 Madrid. 